\title{
DECOMPOSABILITY OF POLYTOPES
}

\author{
Krzysztof Przesławski \& David Yost
}

\begin{abstract}
We reformulate a known characterization of decomposability of polytopes in a way which may be more computationally convenient, and offer a more transparent proof. We apply it to give new sufficient conditions for indecomposability of polytopes, and then illustrate them with some examples.
\end{abstract}

\section{INTRODUCTION}

This paper is concerned with criteria for the indecomposability of polytopes. We recall that a polytope $P$ is decomposable if it is equal to a Minkowski sum $Q+R$ of two polytopes $Q$ and $R$ which are not homothetic to $P$. Linguistic logic forces us to describe all other polytopes as indecomposable. The concept of decomposability is due to Gale [1] although he used a different name. It also makes sense for more general convex bodies, but we will not consider them here. It is not surprising to learn [1] that triangles are indecomposable, and conversely, that any 2-dimensional polygon is the sum of triangles and segments. Gale also announced that any pyramid, i.e. the convex hull of a facet and a single point, is indecomposable. Shephard made perhaps the next serious study of it, showing amongst other things that a polytope is indecomposable if all of its 2-faces are triangles [5, (13)]. Since then, a number of papers have found progessively weaker sufficient conditions for indecomposability and we are continuing this tradition.

The latter result is a special case of [5, (12)], which asserts that a polytope is indecomposable if there is an edge to which all vertices are connected by a strong chain of indecomposable faces. A simple reformulation of this statement is that a polytope is indecomposable if there is a strong chain of indecomposable faces which contains all the vertices.

By a strong chain of faces is meant a finite sequence of faces in which each successive pair shares an edge. McMullen [3] showed that the hypothesis of Shephard's result could be relaxed in the following way: the union of 
this chain need contain only one vertex from each facet, not all of them. His proof, like Shephard's, was geometric in character, although the statement of the hypothesis is graph theoretic. By that, we mean that the 1-skeleton of a polytope is clearly a graph, and the hypothesis is just a statement about this graph.

Earlier Kallay [2] had weakened the hypothesis in several other ways. One was to consider collections of vertices which did not necessarily form a face. For example, three vertices can be pairwise adjacent, whilst their centroid is an interior point of the polytope. (Blissfully unaware of [2], the second author used a similar approach in [7] for examining irreducibility of centrally symmetric polytopes.) Another weakening was to show that each successive pair of the chain could share just two vertices, not necessarily an edge. He adopted a strictly graph theoretic approach, defining the concept of indecomposability for geometric graphs, and showing that a polytope is indecomposable if and only if its 1-skeleton is indecomposable in this sense.

Our aim is to extend some of the results obtained in these works. Although similar to [2], our approach is simpler and more general; in particular, we require no knowledge of spherical complexes. Implicit in [2] is the use of a mapping from the vertices of the polytope into the ambient space. This was explicit in [7] and we carry on with it here.

\section{Basic notions}

All graphs considered here are assumed to have a finite number of vertices. Let $G=(V, E)$ be a graph with set of vertices $V$ and set of edges $E \subset$ $\left(\begin{array}{c}V \\ 2\end{array}\right)$. Let $f, g \in\left(\mathbf{R}^{d}\right)^{V}$. Mostly we will be interested in the 1-skeleton of a polytope, but it is practical to consider this more abstract situation. Let $f, g \in\left(\mathbf{R}^{d}\right)^{V}$. Let $I$ be a non-empty subset of $\mathbf{R}$. We say that $g$ is edgewise $I$-dominated by $f$ if for any pair $u, v \in V$ of adjacent vertices there exists $\alpha \in I$ such that

$$
g(u)-g(v)=\alpha(f(u)-f(v)) .
$$

In the case $I=\mathbf{R}$, we say simply that $g$ is edgewise dominated by $f$, and write $g \preceq f$. (In case $f$ is the identity mapping, $g$ is an isomorphism and $I=(0, \infty)$, this coincides with the concept of local similarity defined in [2].) If $I$ is a non-zero singleton, then we also say that $g$ is similar to $f$.

Observe that the sets $E(f):=\{g: g \preceq f\}$ and $S(f):=\{g: g$ is similar to $f\}$ are vector spaces. Clearly $S(f)$ is the direct sum of the $d$-dimensional subspace of translations and the 1-dimensional subspace of multiples of the 
identity. If these spaces are equal, then $f$ is said to be indecomposable. Thus the quotient space $D(f):=E(f) / S(f)$ relates to "decomposability" of $f$. The dimension of $D(f)$ will be called the index of decomposability of $f$. We denote this index by $\operatorname{dec} f$. Hence $f$ is indecomposable if and only if $\operatorname{dec} f=0$. These notions find a natural interpretation when we discuss decomposability of polytopes.

Suppose that a function $\varphi: V \rightarrow \mathbf{R}$ is given. We say that $\varphi$ attains a (local!) maximum at $v \in V$ if for any $u$ adjacent to $v$ we have $\varphi(v) \geq \varphi(u)$. The set of all maximizers of $\varphi$ is denoted throughout by $\operatorname{argmax} \varphi$.

We begin with an auxiliary result.

LEMma 1 Let $V$ be the set of vertices of a graph $G$. Let $f, g \in\left(\mathbf{R}^{d}\right)^{V}$, and let $g$ be edgewise $(0,+\infty)$-dominated by $f$. Then for any $y \in\left(\mathbf{R}^{d}\right)^{*}$

$$
\operatorname{argmax} y \circ g=\operatorname{argmax} y \circ f \text {. }
$$

Proof. If $v \notin \operatorname{argmax} y \circ f$, then there exists a vertex $w$ adjacent to $v$ such that $y \circ f(v)<y \circ f(w)$. By our assumptions, there exists $\alpha>0$ such that $\alpha(f(w)-f(v))=g(w)-g(v)$. Applying $y$ to this equation, we get readily from the linearity of $y$ that $y \circ g(w)-y \circ g(v)>0$. Thus $v \notin \operatorname{argmax} y \circ g$. The symmetrical relationship between $f$ and $g$ completes the proof.

Recall that a graph $G$ is called a cycle if $|V|=k \geq 3$ and $V$ can be ordered as $\left\{x_{1}, \ldots, x_{k}\right\}$, so that $E=\left\{\left\{x_{1}, x_{2}\right\}, \ldots\left\{x_{k-1}, x_{k}\right\},\left\{x_{k}, x_{1}\right\}\right\}$. The number $k$ is said to be the length of the cycle. The following result is simple, but it does lead us to new examples of indecomposable polytopes.

Proposition 2 Let $C_{k}$ be a cycle of length $k$. Let $f: C_{k} \rightarrow \mathbf{R}^{d}$ be an injection for which $f\left(C_{k}\right)$ is an affinely independent set, that is, elements of $f\left(C_{k}\right)$ are vertices of a simplex. Then $\operatorname{dec} f=0$.

Proof. Let $g \preceq f$. For each $i \leq k$, let $u_{i}=g\left(x_{i+1}\right)-g\left(x_{i}\right)$ and $v_{i}=$ $f\left(x_{i+1}\right)-f\left(x_{i}\right)$ (we let here $x_{k+1}=x_{1}$ ). By definition, for each $i$ there exists $\alpha_{i}$ such that $u_{i}=\alpha_{i} v_{i}$. From $\sum u_{i}=0$, we obtain $\sum \alpha_{i} v_{i}=0$. This equation and the fact that elements $x_{i}$ are affinely independent readily imply that all numbers $\alpha_{i}$ are equal.

We will not make any use of the next result. However we include it, as it helps to understand the situation.

Proposition 3 Let $G=(V, E)$ be a graph. If $|V|>2$ and there exists an injection $f: V \rightarrow \mathbf{R}^{d}$ such that $\operatorname{dec} f=0$, then $G$ is 2-connected. 
Proof. It is clear that $G$ is connected. If $G$ were not 2-connected, then there would exist an edge $\{u, v\}$ whose removal would disconnect the graph. Let $A$ and $B$ be the components of $u$ and $v$ respectively. Defining the function $g$ by $g_{\mid A}=f_{\mid A}$ and $g_{\mid B}=f_{\mid B}+f(v)-f(u)$, it is clear that $g \in E(f) \backslash S(f)$. Consequently, $\operatorname{dec} f \neq 0$.

\section{Decomposability AND Indecomposability.}

A convex polytope $P$ in $\mathbf{R}^{d}$ is said to be decomposable if there exist convex polytopes $Q$ and $R$, which are not non-negative homothets of $P$, such that $P=Q+R$. Otherwise, $P$ is said to be indecomposable.

We use standard notation which will not surprise anyone. By vert $P$ we mean the set of vertices of $P$. A set $F \subset P$ is a face of $P$ if there exists $y \in$ $\left(\mathbf{R}^{d}\right)^{*}$ such that $F=\{v \in P: y(v)=h(P, y)\}$, where $h(P, y)=\max y(P)$. The mapping $y \mapsto h(P, y)$ is called the support function of $P$. We mean by the 1-skeleton of $P$ the graph $G_{P}=(V, E)$ such that $V=\operatorname{vert} P$ and $E$ consists of all these pairs $\{u, v\}$ for which the line segment $[u, v]$ is a 1-dimensional face of $P$.

As an immediate consequence of Lemma 1 we have

Lemma 4 Let $G_{P}$ be the 1-skeleton of a polytope $P$. For $y \in\left(\mathbf{R}^{d}\right)^{*}$, let $C=\{v \in \operatorname{vert} P: y(v)=h(P, y)\}$. If $g: \operatorname{vert} P \rightarrow \mathbf{R}^{d}$ is edgewise $(0, \infty)$ dominated by $\operatorname{id}_{\mathrm{vert} P}$, then $g(C)$ is equal to

$$
\{w \in g(\operatorname{vert} P): y(w)=\max y \circ g(\operatorname{vert} P)\} .
$$

Proof. It suffices to observe that $C=\operatorname{argmax} y \circ \mathrm{id}_{\text {vert }} P$.

Let conv A denote the convex hull of $A \subset \mathbf{R}^{d}$. Let $Q=\operatorname{conv} g(\operatorname{vert} P)$, where $g$ is as in the lemma. It follows that $g$ is a one-to-one correspondence between vert $P$ and vert $Q$ and that $g^{-1}$ is edgewise $(0, \infty)$-dominated by $\operatorname{id}_{\text {vert } Q}\left(g^{-1}\right.$ relates here to the 1 -skeleton of $\left.Q\right)$. Moreover, the induced mapping $\widetilde{g}$ defined on faces of $P$ by the formula

$$
\widetilde{g}(F)=\operatorname{conv} g(F)
$$

is an isomorphism of the facial structures of $P$ and $Q$.

COROLlary 5 If the mapping $g$ is edgewise $[0, \infty)$-dominated by $\operatorname{id}_{\mathrm{vert} P \text {, }}$, then for every face $F$ of $P$ and every $y \in\left(\mathbf{R}^{d}\right)^{*}$ such that $y(v)=h(P, y)$, whenever $v \in F$,

$$
g(\operatorname{vert} F) \subset\{w \in g(\operatorname{vert} P): y(w)=h(Q, y)\},
$$


where again $Q=\operatorname{conv} g(\operatorname{vert} P)$.

Proposition 6 If $g$ is edgewise $[0,1]$-dominated by $\operatorname{id}_{\mathrm{vert}} P$, then $Q$, defined as before, is a summand of $P$, that is, there exists a polytope $R$ such that $P=Q+R$.

Proof. Let $k(u)=u-g(u)$. The function $k$ is also edgewise $[0,1]$-dominated by $\operatorname{id}_{\operatorname{vert}} P$. Let $R=\operatorname{conv} k(\operatorname{vert} P)$. For $y \in\left(\mathbf{R}^{d}\right)^{*}$, choose $v \in \operatorname{vert} P$ such that $y(v)=h(P, y)$.

By the preceding corollary, $y \circ g(v)=h(Q, y)$ and $y \circ k(v)=h(R, y)$. Moreover, by the definition of $k, y(v)=y \circ g(v)+y \circ k(v)$. Thus, $h(P, y)=$ $h(Q, y)+h(R, y)$, which implies $P=Q+R$.

The next theorem is essentially [2, Corollary 5]. It is formulated there in a different but equivalent form.

TheOrem $7 P$ is decomposable if and only if $\operatorname{dec}_{\mathrm{id}} P \neq 0$. Moreover, any function $g$ that is edgewise $[0,1]$-dominated by $\mathrm{id}_{\mathrm{vert}} P$ and the same time not similar defines a non-homothetic summand of $P$.

Proof. If $P$ is decomposable, there exist polytopes $Q$ and $R$, which are nonhomothetic to $P$, such that $P=Q+R$. Thus, for any $v \in \operatorname{vert} P$ there exists a unique element $g(v) \in \operatorname{vert} Q$ for which we have $v \in g(v)+R$. It is easy to see that $g$ is edgewise $[0,1]$-dominated by $\operatorname{id}_{\text {vert } P \text {. Since } g \text { is onto }}$ vert $Q$ and $Q=\operatorname{conv} g($ vert $P), g$ can not belong to $S\left(\operatorname{id}_{\text {vert } P}\right)$, for otherwise $Q$ would be a homothetic copy of $P$.

Conversely, suppose that $\operatorname{decid}_{\text {vert } P} \neq 0$. Then there exists some $f \in$ $E\left(\operatorname{id}_{\text {vert } P}\right) \backslash S\left(\operatorname{id}_{\text {vert } P}\right)$. If $\alpha>0$ is sufficiently small, $\alpha f$ will be edgewise

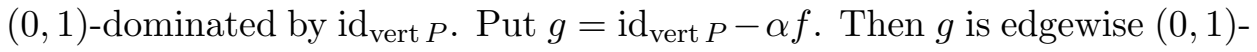
dominated by but not similar to $\operatorname{id}_{\text {vert }} P$. By Proposition $6, Q=\operatorname{conv} g(P)$ is a summand of $P$. The fact that it is not a homothetic copy of $P$ is clear.

The second part of the theorem is rather obvious.

For further use we shall need a graph theoretic consequence of the above result, essentially [2, Proposition 8]. We note that the subgraph $G$ here need not be the 1-skeleton of any polytope.

Theorem 8 Let $P$ be a d-dimensional polytope in $\mathbf{R}^{d}$. Then $P$ is indecomposable if and only if there exists a subgraph $G=(V, E)$ of the 1-skeleton of $P$ such that $\mathrm{id}_{V}$ is indecomposable (as a mapping related to $G$ ), and $V$ meets every facet of $P$. 
Proof. We have to show the 'if' part only, as the 'only if' part is a consequence of the preceding theorem. (It suffices to let $G$ be the 1-skeleton of P.)

Suppose that $Q$ is a nontrivial summand of $P$, that is $Q$ contains more than one element. Take the function $g: \operatorname{vert} P \rightarrow \operatorname{vert} Q$ defined as in the preceding proof. Since $\operatorname{id}_{V}$ is indecomposable, there is a number $\alpha$ and a vector $x \in \mathbf{R}^{d}$ such that

$$
g_{\mid V}=\alpha \operatorname{id}_{V}+x .
$$

It is clear that shifting $Q$ if necessary we may assume $x=0$. We may also assume that 0 belongs to the interior of $P$.

Let $y \in\left(\mathbf{R}^{d}\right)^{*}$ be any outer normal of a facet $F$ of $P$ and let $v \in V \cap F$. By (1) and Corollary 5

$$
h(Q, y)=y \circ g(v)=\alpha y(v)=\alpha h(P, y) .
$$

Obviously, for at least one of the normals we have $h(Q, y)>0$. Hence $\alpha>0$ and $h(Q, y)=h(\alpha P, y)$ for each normal $y$. Since $Q$ is a summand of $P$, we deduce that $Q=\alpha P$ which implies the indecomposability of $P$.

Our next notion relates to the notion of strongly connected family of polytopes which is useful in formulating sufficient conditions for indecomposability (see $[3,6,7])$.

Let $\mathcal{G}$ be a family of subgraphs of a graph $G$. We say that $\mathcal{G}$ is strongly connected if for any pair of graphs $G, K \in \mathcal{G}$, there exists a sequence $G_{1}, \ldots, G_{k}$ of graphs in $\mathcal{G}$ with sets of vertices $V_{1}, \ldots, V_{k}$, respectively, such that $G_{1}=G, G_{k}=K$ and $\left|V_{i} \cap V_{i+1}\right| \geq 2$ for $i=1, \ldots k-1$. Such a sequence will be called a strong chain of graphs.

Now, as a simple consequence of the previous result we obtain

TheOREM 9 Let $P$ be a polytope in $\mathbf{R}^{d}$. Let $\mathcal{G}$ be a strongly connected family of subgraphs of the 1-skeleton $G_{P}$. If for each $(V, E) \in \mathcal{G}$ the identity map $\mathrm{id}_{V}$ is indecomposable and $W:=\bigcup\{V:(V, E) \in \mathcal{G}\}$ meets every facet of $P$, then $P$ is indecomposable.

Proof. Let $D=\bigcup\{E:(V, E) \in \mathcal{G}\}$. It suffices to show that $\mathrm{id}_{W}$, as a mapping related to $G:=(W, D)$, is indecomposable. Let $g: W \rightarrow \mathbf{R}^{d}$ be similar to $\operatorname{id}_{W}$. Fix $u \in W$. For any $w \in W$ there exists a strong chain $G_{1}, \ldots, G_{k}$ of graphs in $\mathcal{G}$ such that $u \in V_{1}$ and $w \in V_{k}$. Let $g_{i}$ be the restriction of $g$ to $V_{i}$. By our assumptions, for each $i$ there exist $\alpha_{i} \in \mathbf{R}$ and 
$z_{i} \in \mathbf{R}^{d}$ such that $g_{i}(x)=\alpha_{i} x+z_{i}$. By the definition of a strong chain, there exist two different elements $s$ and $t$ which belong to $V_{i} \cap V_{i+1}$. Therefore,

$$
g(s)-g(t)=\alpha_{i}(s-t)=\alpha_{i+1}(s-t),
$$

which implies that $\alpha_{i}=\alpha_{i+1}$ and also $z_{i}=z_{i+1}$. In consequence, $g$ is similar to $\operatorname{id}_{W}$.

Previous workers $[3,4,5,6]$ have usually assumed that each graph $(V, E)$ belonging to $\mathcal{G}$ has its vertices $V$ contained in a proper face of the polytope $P$. We emphasize that this assumption is not necessary. This point is implicit in [2] and explicit in [7, p. 137], although the latter deals only with triangles.

Applicability of Theorem 9 depends on the existence of a reasonable class of graphs embedded into $\mathbf{R}^{d}$ for which the identity is indecomposable. As is shown by Proposition 2, the simplest graphs that conform to these demands are cycles. We shall make use of the following

Corollary 10 Let $P$ be a polytope in $\mathbf{R}^{d}$. Let $\mathcal{G}$ be a strongly connected family of subgraphs of the 1-skeleton $G_{P}$. If each $(V, E) \in \mathcal{G}$ is a cycle with an affinely independent set of vertices and each facet of $P$ has a vertex that belongs to a certain graph from $\mathcal{G}$, then $P$ is indecomposable.

\section{Some applications}

Meyer [4] and Kallay [2] gave examples of decomposable 3-dimensional polytopes possessing combinatorially equivalent copies which are indecomposable. It is known [6, p. 47] that any such polytope must have at least eight vertices. Kallay's polytope has ten vertices while Meyer's has even more. Smilansky [6, Theorem 6.11(b)] announced the existence of a 3-dimensional polytope of this kind with exactly eight vertices, and referred the reader to his thesis for the details. As an application, we give now an example of this kind. We have not had access to Smilanky's thesis but we would not be surprised if his example is equivalent to ours.

EXAMPLE 11 A conditionally decomposable polyhedron with eight vertices.

Let $P$ be the convex hull of the following points: $A_{1}=(2,1,0), A_{2}=$ $(1,2,0), A_{3}=(-2,-1,0), A_{4}=(-1,-2,0), B_{1}=(-1,-1,1), B_{2}=$ $(1,1,1), C_{1}=(1,1,-1)$ and $C_{2}=(-1,-1,-1)$. It is easy to see that 
$P$ is just the sum of the standard octahedron and a segment parallel to $(1,1,0)$, i.e. the line segment $\left[C_{1}, C_{2}\right]$ is a summand of $P$. Next we define another polytope $Q$, whose vertices are labeled in the same way as for $P$. We replace $A_{1}$ by $(2+3 \varepsilon, 1,3 \varepsilon)$ and replace $B_{2}$ by $(1+2 \varepsilon, 1,1+2 \varepsilon)$ (where $\varepsilon$ need not be so small), and let the other vertices be the same as in $P$. Thus $Q$ is obtained simply by perturbing two vertices of $P$. It can be easily verified that the labeling induces a one-to-one correspodence between the facial structures of $P$ and $Q$. In detail, $A_{1}$ is still in the plane $x-y-z=1$, $B_{2}$ is still in the plane $x-y-z=-1$ and both of them are in the plane

$$
(\varepsilon-1) x+(\varepsilon+1) y-(\varepsilon+1) z=-1-3 \varepsilon,
$$

as are the original points $A_{4}$ and $B_{1}$. So $Q$ is combinatorially equivalent to $P$. Then for $Q$, the cycle $A_{1} A_{2} A_{3} A_{4}$ is a subgraph of the 1 -skeleton of $Q$ which satisfies the assumptions of Corollary 10; in particular, the vertices $A_{i}$ are affinely independent. Consequently, $Q$ is indecomposable.

The point of this note is that there are other polytopes which can be shown to be indecomposable by Corollary 10 but not by earlier results. We present some now.

EXAMPLE 12 There is a combinatorially indecomposable polyhedron with 11 vertices and 6 triangular faces, no two of which have a common edge. Thus traditional methods of proving indecomposabilty are not available. But in any geometric realization, it has two affinely independent 4-cycles, with two vertices in common, whose union touches every face.

Put $A=(1,0,-1), B=(0,-1,1), C=(-1,1,0), D=(1,-1, h)$, $E=(1,-1,-h), F=(-1, h, 1), G=(-1,-h, 1), H=(h, 1,-1), J=$ $(-h, 1,-1), N=(1,1,1)$ and $S=(-1,-1,-1)$. If one looked "down" on the resulting polytope, one would see that the faces containing $N$, in clockwise order, are $A N D E, B D N, B F G N, C F N, C J H N$ and $A H N$. Looking "up", one would see that the faces containing $S$, in clockwise order, are $A E S, A H J S, C J S, C S F G, B G S$ and $B D E S$. So no two triangular faces have a common edge.

Still, indecomposabilty can be proved easily by noting that the connected 4-cycles $N A S B$ and $N B S C$ are affinely independent and their union touches every face.

There exist polytopes combinatorially equivalent to this one, in which the corresponding 4-cycle $N A S B$ is affinely dependent. (A concrete example is given after Example 13.) Nevertheless, this polytope is combinatorially 
indecomposable. It suffices to observe that of the three connected 4-cycles $N A S B, N A S C$ and $N B S C$, at least two must be affinely independent (in any given geometric realization). Otherwise the vertices $A, B, C, N$ and $S$ would be co-planar.

Note that any indecomposable polyhedron must have at least four triangular faces [6, Corollary 6.8].

EXAMPLE 13 There is a combinatorially indecomposable polytope with nine vertices and only four triangular faces, of which no two have a common edge.

The same argument using Corollary 10 works but this time it is simpler; we need consider only one 4-cycle, not two. Let $A=(-1,1,-1)$, $B=(1,1,-1), C=(1,-1,-1), D=(-1,-1,-1), E=(-1,1,0), F=$ $(-1,1 / 2,3 / 4), G=(1,-1 / 2,3 / 4), H=(1,-1,0)$ and $J=(0,0,1)$, and let $P$ be their convex hull. We now list the faces of $P$, together with the equations of the planes containing them.

$$
\begin{array}{r}
A B C D, z=-1, \quad A D E F, x=-1, \quad B C G H, x=1, \\
B E F J, x+3 y+2 z=2, \quad D G H J,-x-3 y+2 z=2, \\
A B E, y=1, \quad C D H, y=-1, \\
B G J, 5 x+7 y+6 z=6, \quad D F J,-5 x-7 y+6 z=6 .
\end{array}
$$

In any polyhedron equivalent to $P$, the 4-cycle $B C D J$ must be affinely independent. It clearly touches every face, so $P$ is indecomposable. Again, arguments with triangles will not work.

Let us remark that if we add two extra vertices to the polyhedron above, $K=(-1 / 2,0,-2)$ and $L=(1 / 2,0,-2)$, then the resulting polyhedron is combinatorially equivalent to Example 12 . 


\section{References}

[1] D. Gale, Irreducible Convex Sets, Proc. International Congress of Mathematicians Amsterdam, 1954, vol. 2, 217-218.

[2] M. Kallay, Indecomposable Polytopes, Israel J. Math. 41 (1982), 235243.

[3] P. McMullen, Indecomposable Convex Polytopes, Israel J. Math. 58 (1987), 321-323.

[4] W. J. Meyer, Indecomposable Polytopes, Trans. Amer. Math. Soc. 190 (1974), 77-86.

[5] G. C. Shephard, Decomposable Convex Polytopes, Mathematika 10 (1963), 89-95.

[6] Z. Smilansky, Decomposability of Polytopes and Polyhedra, Geom. Dedicata 24 (1987), 29-49.

[7] D. Yost, Irreducible Convex Sets, Mathematika 38 (1991), 134-155.

52B11: CONVEX AND DISCRETE GEOM-

ETRY: Polytopes and polyhedra;

n-dimensional polytopes.

Faculty of Mathematics, Computer Science and Econometrics, University of Zielona Góra, 65-246 Zielona Góra, POLAND

e-mail: K.Przeslawski@im.uz.zgora.pl

School of Information Technology and Mathematical Sciences, University of Ballarat, PO Box 663,

Ballarat, Vic. 3353

AUSTRALIA

e-mail: d.yost@ballarat.edu.au

Fax: * 61353279289 